\title{
Measuring the Contextual Dimension of User Experience: Development of the User Experience Context Scale (UXCS)
}

\author{
Carine Lallemand \\ Industrial Design Department, Eindhoven University of \\ Technology, Eindhoven, the Netherlands; HCI Research \\ Group, University of Luxembourg, Esch-sur-Alzette, \\ Luxembourg \\ c.e.lallemand@tue.nl
}

\begin{abstract}
The context of use has been highlighted for a long time as being a key factor impacting User Experience (UX). Yet current UX evaluation tools, especially questionnaires, rarely encompass an explicit investigation of the context. With the ever-growing trend for mobile products and ubiquitous computing, the absence of a dedicated measurement tool becomes critical. Based on a review of relevant literature and a fine-grained categorization of contextual factors, we developed the UX Context Scale (UXCS), a 30-items instrument allowing for a measure of context properties, as perceived by the user. We report on the development of the scale and present a first validation study $(N=137)$. A principal component analysis on the subjective items reveals a 6-components structure: Physical Context, Social Context, Internal Context, Perceived Resources, Task Context, and Temporal Context. Reliability of each subscale is high and further analyses confirm the relevance of this tool for UX evaluation.
\end{abstract}

\section{CCS CONCEPTS}

- B7; Human-centered computing $\rightarrow$ Human computer interaction (HCI); HCI design and evaluation methods.

\section{KEYWORDS}

User experience, context of use, scale development, UX evaluation, UX Context Scale

\section{ACM Reference Format:}

Carine Lallemand and Vincent Koenig. 2020. Measuring the Contextual Dimension of User Experience: Development of the User Experience Context Scale (UXCS). In Proceedings of the 11th Nordic Conference on HumanComputer Interaction: Shaping Experiences, Shaping Society (NordiCHI '20), October 25-29, 2020, Tallinn, Estonia. ACM, New York, NY, USA, 13 pages. https://doi.org/10.1145/3419249.3420156

\section{INTRODUCTION}

User experience (UX) is commonly described as the holistic quality of the interaction between a user and an interactive system. Even if no clear consensus has been reached on the definition of UX

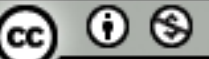

This work is licensed under a Creative Commons Attribution-NonCommercial International 4.0 License.

NordiCHI '20, October 25-29, 2020, Tallinn, Estonia

(c) 2020 Copyright held by the owner/author(s).

ACM ISBN 978-1-4503-7579-5/20/10.

https://doi.org/10.1145/3419249.3420156

\author{
Vincent Koenig \\ HCI Research Group, University of Luxembourg, \\ Esch-sur-Alzette, Luxembourg \\ vincent.koenig@uni.lu
}

[33], UX researchers and practitioners agree on the three classical pillars influencing UX: the user, the system and the context [50]. Following this common understanding, Hassenzahl and Tractinsky [18] define UX as: "A consequence of a user's internal state (predispositions, expectations, needs, motivation, mood, etc.), the characteristics of the designed system (e.g., complexity, purpose, usability, functionality, etc.) and the context (or the environment) within which the interaction occurs (e.g., organizational/social setting, meaningfulness of the activity, voluntariness of use, etc.)" (p. 95). Classically considered as a crucial factor in the fields of Ergonomics and Human-Computer Interaction (HCI), the notion of context is almost always mentioned in UX frameworks or models. The "situatedness of action" [37] and the acknowledgment of a close interaction between action and situation during the interaction with technology contribute to highlight the central role played by the context. Numerous studies have evaluated interactive systems in specific contexts of use, thereby showing what challenges are set by these contextual factors for the interaction [32, 46, 47].

While UX assessment questionnaires tend to focus on the perceived qualities of a product or system [19], or the emotions triggered [41], little attention is paid to the explicit measure of both objective and perceived context of use. One may argue that this dimension is studied upstream of a project, with more qualitative methodologies (e.g., contextual inquiry or design probes) or through sensor technology. However, since online UX studies become a widespread tool, a measure of perceived context "quality" grows even more important. Understanding and assessing the influence of contextual factors in UX thus remains a crucial endeavour for the design of interactive products. The present study attempts to address this challenge. Our contribution is threefold: (a) we review and discuss theories on the notion of context and its underlying dimensions (b) we introduce the UX Context Scale (UXCS) and the rationale behind its development. We describe the validation of the UXCS through an online study involving 137 participants. (c) Through this case study, we also provide an illustrative analysis of the links between contextual factors and UX. The paper concludes with the implications of this research for UX evaluation.

\section{RELATED WORK}

\subsection{User Experience is Contextual}

According to the Oxford dictionary lexico.com, context is generally defined as 'the circumstances that form the setting for an event, statement, or idea, and in terms of which it can be fully understood'. An experience, whatever form it takes, is indeed inevitably embedded 
in a specific setting and should therefore be considered as "coloured" by a specific context. User experiences, being specific categories of experiences "derived from encountering systems" [50], are no exceptions to this rule. The results of a UX survey conducted in 2008 [36] and replicated in 2012 [33], confirm this observation by showing that UX professionals mainly agree on the fact that " $U X$ occurs in and is dependent on the context in which the artefact is experienced".

From a theoretical point of view, UX is contextual per nature: the field of UX emerged out of several theories highlighting the essential role of the context. Situated action [62] which focuses on the understanding of human acts in context, is one of them. Traditional User-Centred Design, another deep root of UX, also relies on the requirement to "understand and specify the context of use" [23]. The majority of UX models, whether theoretical or more pragmatic, include the context of use as one of the main factors impacting UX [7, 12, 18, 23, 39, 61]. The UX White Paper [50], states that "UX may change when the context changes, even if the system does not change" (p. 10). Numerous field studies confirm this statement $[32,67]$.

Along the same lines, context-aware computing is a field of research and practice introduced by Schilit, Adams and Want [58] to describe devices that may sense several parameters of the environment, use the situation and react accordingly to the situation. As context awareness has mainly been used for applications where the user's context is rapidly changing (e.g., handheld) [9], user location was one of the first parameters of interest. Many others followed as the notion of context was conceptualized in $\mathrm{HCI}$.

\subsection{What is the Context in HCI?}

Following Dourish's paper on the role of context [11], it is essential to understand "what we talk about when we talk about context". The notion of context in HCI has indeed been defined in various ways. Major contextual models or taxonomies were first built to support the specification of the use context for usability studies $[1,4,31,38,40]$, and subsequently extended beyond the scope of usability to that of user experience.

The ISO standard 9241-11 [25] identifies users, tasks, equipment and environment as the first-level attributes, further broken down into sub-attributes and categories given as a set of examples. Users encompass user types, skills, knowledge and personal attributes. Equipment is subdivided into basic description and specification. Environment includes three categories, organizational, technical and physical environment. Maguire [38] provides a description of the main contextual aspects to be considered in a context of use analysis. His first-level components include user goals and characteristics, tasks, technical environment, physical environment and finally social or organizational environment. Similarly, Alonso-Rios et al. [1] provided a context-of-use taxonomy for usability studies, where the classical triad user, task and environment constitutes the hierarchy's first-level. The taxonomy aimed at lessening the tendency to overlook characteristics of the context, which the authors believed to be caused by the lack of a clear and comprehensive taxonomy including precise definitions of context of use components.

Classifications of contextual dimensions, or context models, were also produced in the field of context-awareness. These simplified representations of context intend to structure or describe contextual elements [3]. Dey and Abowd [9] proposed the distinction between four contextual dimensions, namely location, identity, activity and time. Kaltz, Ziegler and Lohmann [30] differentiated three categories of context: user and role, process and task and finally time and device. However, following Dey's [9] definition of the context as "any information that can be used to characterize the situation of an entity," more thorough and multidisciplinary models of the context of use needed to be explored in order to contribute to the understanding of user experience. Bradley and Dunlop [5] proposed a multidisciplinary model of context of use integrating $\mathrm{HCI}$ and context-aware computing perspectives. They define context of use as "anything that influences the process in which focal user actions are undertaken" and suggest the following dimensions:

- Task context: people and objects surrounding the task, which offer advantages ("resources") and drawbacks ("constraints") for its achievement.

- Physical context: includes the environmental location, nearby physical elements and their attributes, but also weather and light conditions.

- Social context: influences of surrounding people (relationship, dialogue, presence and behaviour).

- Temporal context: influences of past experiences in terms of expectations, and explanations of the present. It also includes higher-level temporal context (e.g. current time, date or season).

- Application context: the information flow between the user and the device (e.g. representation of the application's state and functioning).

- Cognitive context: the user's cognitive abilities, habits and attitudes.

The authors [5] also distinguish two contextual properties: meaningful and incidental. Meaningful context is related to the achievement of the user's higher-level goals, and is selected by the user. Incidental context describes, "incidental occurrences in the contextual world ... e.g., bumping into a friend", which will exert an influence on the user. Finally, they considered user activity as a process in which cognitive goals are "continually shaped by people's perception of the meaningful and incidental contextual worlds" (p. 428), while at the same time user actions to fulfil these goals contribute to shaping future context.

More recently, several authors have reviewed context models to provide a consolidated view on context dimensions. A literature review by Jumisko-Pyykkö and Vainio [27] summarises the most cited elements of context of use, as described in 109 articles from HCI venues. Six most studied components of context were identified, presented in the following according to the percentage of articles referring to each component:

- Social context $(66,1 \%)$ : influence of others, present physically or in a technology-mediated fashion. Social context also includes social norms, values, and attitudes.

- Physical context (61.5\%): spatial location, functional space, and place. Includes where the user is located, elements (artefacts) of the near environment they could interact with and general functions and attributes of their surroundings. 
- Technical and informational contexts (36.7\%): technical aspects such as available services and applications, interoperability, type of device, and mixed reality.

- Temporal context (35.8\%): duration of the interaction, time of day and date (in relation to user activity patterns), level of time pressure, and synchronism of the task.

- Task context (31.2\%): task interruptions (attentional shift away from the task), number of different competing tasks (multitasking) as well as task's category (goal-oriented or action-oriented)

- Transitions (20,2\% - 22/109 papers): Some cases (e.g., a mobile context of use) also involve a changing environment, which results in "transitions" between the dimensions of context.

It should be noted that Jumisko-Pyykkö and Vainio [27] do not detail the internal context of the user as a proper context dimension. This tendency to isolate the user as a distinct entity might result from the aim of reducing the overload upon the term context as suggested by ISO standards [23, 24]. In their analysis of 36 context models and 3741 unique context elements, Bauer and Novotny [3] however consider the user as one of the three main component of the social context along with the social environment and the activity. A user's identity includes both a stable profile (e.g., demographics) and a situational profile, which varies more frequently (e.g., psychographics such as mood, affect, preferences, knowledge).

Knowing which contextual dimensions are involved in humancomputer interactions is a first step toward the understanding of how context impacts UX. While there are some differences in the number of dimensions or labelling, contextual models and taxonomies globally agree on the main dimensions to take into account when studying the context. Social context, physical context, technical context, temporal context, internal context, and finally task context appear to be the most relevant factors impacting the interaction. Transitions might also occur when the environment is changing during the course of the interaction.

\subsection{The Context in UX Questionnaires}

Questionnaires appear to be a good vector for contextual studies, allowing users to answer them during or just after use in their ecological setting. However, remote studies or self-declared measures will require specific contextual-aspects measurement tools. Existing UX assessment self-administered questionnaires are predominantly focused on pragmatic and hedonic qualities of interactive systems without actually including the context as a variable to be explicitly measured. The AttrakDiff scale [19] or the User Experience Questionnaire [35] are two examples of "product-centred" UX measurement tools. Their underlying models might take context into account, assuming that the evaluation after exposure to a product always implies and includes a certain context, yet product and context are not studied separately and the resulting perception is an evaluation of the product in context.

Other questionnaires may be used as a complement to these measures. As an example, some authors [64] report the use of three generic questions about the context extracted from the Geneva Appraisal Questionnaire [57]: when, where, and with whom a reported user experience took place. Partala and Kallinen [48] used a self-made 10 statements questionnaire studying the context of the user experience. It was specifically developed for their experiment based on the context framework by Jumisko-Pyykkö and Vainio [27] and included the most cited contextual dimensions (temporal, physical, social, task and technical - contexts).

Regarding more specific aspects of the context, numerous measurement tools, primarily developed by psychologists, focus on the internal context of the user, namely the affects [52, 66], emotions $[6,26,57]$, psychological needs [59] or intrinsic motivation [53]. Amongst the questionnaires commonly used to study UX, only a few address the notion of social context and those are predominantly used in the context of games [43]. Finally, to the best extent of our knowledge, no existing UX questionnaire takes explicitly into account a measure of the physical context of the interaction.

\section{DEVELOPMENT OF THE UX CONTEXT SCALE}

\subsection{Objectives of the Scale}

The development of a UX Context Scale (UXCS) aims at providing a holistic measurement of contextual factors for UX assessment. The creation of this tool contributes to filling the gaps in UX evaluation questionnaires, which lack a self-declared measure of the context quality. The measurement of both objective and perceived aspects of the context is especially relevant for controlling the influence of the context. In academia, the UXCS might be used for studying contextual factors impacting UX by providing insights into the interrelationships between contextual dimensions and UX, resulting in actionable recommendations for UX practitioners. In industry, the UXCS scale might be used both for the design of an interactive system or for its evaluation. In the first case, the focus would be on the process (formative evaluation) allowing product designers to better take into account contextual factors when designing a product. In the second case, the focus would be on the product itself (summative evaluation) and the UXCS would collect valuable information on the influence of context on the perceived quality of interaction. Both applications are especially relevant for highly context-dependent products, for example mobile devices [32] [65].

\subsection{Scale Development Process}

Following the best practices on summated rating scale construction [60], we first conducted a literature review to define the dimensions and subdimensions of context relevant for the evaluation of UX. Six main contextual dimensions were identified to compose the UXCS. A fine-grained categorization of contextual dimensions was needed to understand which contextual factors affect user experience and how this occurs. We then designed a first experimental version of the scale under the format of semantic differential items. Each subscale is composed of both objective and perceived contextual aspects. The number of items in each subscale is variable and depends on the importance of each dimension as described in the literature and on the number of subdimensions in each dimension. We submitted the initial pool of items to five HCI experts and checked the scale's face validity by asking them to review each item separately and also to assess if the scale and subscales appeared to be a good measure of the interaction context. These experts were research scientists with a background in psychology active in the field of $\mathrm{HCI}$ and knowledgeable in UX theory and evaluation as well as scale 
Table 1: Description of UXCS subscales (experimental version)

\begin{tabular}{|c|c|c|}
\hline Subscale & Items & Description \\
\hline $\begin{array}{l}\text { Physical context } \\
\text { (PHYS-CTX) }\end{array}$ & 7 & $\begin{array}{l}\text { Focused on some core properties of the physical deployment environment (e.g., noise, temperature } \\
\text { and lighting). From an objective perspective, it also gathers data on the level of familiarity related to } \\
\text { the location and the mobility to know if the user's context is stable or changing rapidly (i.e., level of } \\
\text { dynamism). }\end{array}$ \\
\hline $\begin{array}{l}\text { Social context } \\
\text { (SOC-CTX) }\end{array}$ & $5^{*}$ & $\begin{array}{l}\text { Interactions between the users and other people. Their mere presence will influence users' } \\
\text { perceptions and behaviours. From an objective perspective, this subscale informs whether the } \\
\text { interaction took place in a public or private place, with or without social interactions. The subjective } \\
\text { perspective is focused on the feelings of relatedness and social support [14]. }\end{array}$ \\
\hline $\begin{array}{l}\text { Internal context } \\
\text { (INT-CTX }\end{array}$ & $11^{*}$ & $\begin{array}{l}\text { Refers to the user, especially the situational profile of a user's identity as defined by [3]: their mood } \\
\text { motivation and interest in the system, as well as previous expectations and opinions about the } \\
\text { system. }\end{array}$ \\
\hline $\begin{array}{l}\text { Technical context } \\
\text { (TEC-CTX) }\end{array}$ & 1 & $\begin{array}{l}\text { Asks users whether they have encountered any technical issues with the system, without asking } \\
\text { them to define precisely what type of issue they had to cope with. }\end{array}$ \\
\hline $\begin{array}{l}\text { Task context } \\
\text { (TASK-CTX) }\end{array}$ & $5^{*}$ & $\begin{array}{l}\text { Not focused on tasks per se - as this would imply as many tasks as users - but rather on the task } \\
\text { process and focus devoted to the task. Degree of control, multitasking (competing tasks) and } \\
\text { obstrusiveness (distractions) are of particular interest. }\end{array}$ \\
\hline $\begin{array}{l}\text { Temporal context } \\
\text { (TEMP-CTX) }\end{array}$ & 4 & $\begin{array}{l}\text { Users' perceived temporal aspects of the interaction, illustrated by the level of time pressure or the } \\
\text { adequacy of the moment when it took place. Duration of last interaction and regularity of use are } \\
\text { also explored as objective contextual factors }\end{array}$ \\
\hline
\end{tabular}

* In the final version of the UXCS scale (see Appendix A), the social context includes 4 items, the task context includes 4 items and the internal context is split in two dimensions: internal context and perceived resources.

development. The expert review was first conducted individually, followed by an expert committee meeting. This expert review resulted in a slight decrease in the number of items, as some items did not reach consensus amongst the experts. We also pilot tested the scale on 10 users to ensure the understandability of the items and the format, resulting in changes in the wordings of several items. Users encountered no issue in understanding the item format and reported that the scale overall was easy to understand and easy to use. After slight refinements, we administered the scale to a sample of 147 respondents through an online study.

3.2.1 Contextual Dimensions and Subdimensions. The experimental version of the UX Context Scale was composed of 33 items (Table 1), divided into 6 subscales, as derived from the literature review. After refinement (process described under section 4), the final version of the scale is composed of 30 items (12 items measuring the objective context and 18 items measuring the perceived context) divided into 7 dimensions.

Social, physical, task, temporal and technical context dimensions are part of the core components as identified by Jumisko-Pyykkö and Vainio [27] in their literature review. Regarding the internal context, it has been pinpointed by Korhonen et al. [32] as missing in most context studies since it describes the users' characteristics [27, 51]. Following Dey [10] and [3], users were considered in our study as "entities" in the same way as location or artefacts, and the assessment of their internal state was therefore included as the internal context subscale in the UXCS.

Despite the importance of the technical context involved in the interaction (i.e., the quality of the technical device and environment supporting the interaction), technical-related aspects are very difficult to evaluate from the users' point of view, as users are generally not IT experts. We therefore decided to only ask final users whether they have encountered technical issues with the system, without asking them to define precisely the type of issue. This question also allowed our scale to remain at a generic level, compatible with the assessment of various types of systems or products.

3.2.2 Objective and Subjective Contextual Factors. The UXCS encompasses both objective and subjective (i.e., perceived) parameters of the user's context in each subscale. Objective items have no valence, they are meant to describe factual information (e.g., being in a public or private place). Subjective items are tinted by a value judgment (e.g., being in an unpleasant vs. pleasant location), one extreme of the scale being negative, the opposite being positive. Objective items characterize the situation in which the user is placed and may also be used from a research point of view to assess the impact of specific objective situations on UX. They will therefore be used as informative variables, helpful to interpret users' opinions and feelings toward a system. Subjective items will be averaged per subscale to constitute the evaluation of the perceived context qualities. They will therefore constitute core factors to study. Principal component analyses have been performed on subjective items only to check for the validity of the UXCS structure while reliability has been assessed through separate analyses.

3.2.3 Response Categories and Rating Scales. When designing a measurement scale, the number and type of response categories has to be carefully defined, as it may influence the psychometrical data quality. Standard advice is to use five to nine categories [56]. The UXCS uses 7-points bipolar anchors (called "semantic differentials" 
Table 2: Experimental version of the User Experience Context Scale (UXCS)

\begin{tabular}{|c|c|c|}
\hline \multicolumn{3}{|c|}{ While using the system, I was: } \\
\hline \multicolumn{3}{|c|}{ Physical context } \\
\hline PHYS1 & In an unfamiliar place & In a familiar place \\
\hline PHYS2 & In an unpleasant location & In a pleasant location \\
\hline PHYS3 & In a noisy place & In a quiet place \\
\hline PHYS4 & In a moving / vibrating environment & In a steady environment \\
\hline PHYS5 & Unpleased with the temperature & Pleased with the temperature \\
\hline PHYS6 & Unpleased with the lighting & Pleased with the lighting \\
\hline PHYS7 & Moving (mobile usage) & Remaining still \\
\hline \multicolumn{3}{|c|}{ Social context } \\
\hline SOC1 & In a public space & In a private space \\
\hline SOC2 & Not interacting with people & Interacting with people \\
\hline SOC3 & Feeling alone & Feeling related to other people \\
\hline SOC4 & Feeling unsupported & Feeling supported \\
\hline SOC5* & ${ }^{*}$ Bothered by others & Unbothered by others* \\
\hline \multicolumn{3}{|c|}{ Internal context } \\
\hline INT1 & I had no expectations about the system & I had high expectations about the system \\
\hline INT2 & I had no previous experience with the system & I already had experience with the system \\
\hline INT3 & I had a bad opinion about it & I had a good opinion about it \\
\hline INT4* & ${ }^{*}$ I felt pressed to use it & I felt free to use it $^{*}$ \\
\hline INT5 & Demotivated & Motivated \\
\hline INT6 & Not interested & Interested \\
\hline INT7 & In a bad mood & In a good mood \\
\hline INT8 & I had insufficient skills to use it & I had sufficient skills to use it \\
\hline INT9 & I did not have enough time to spend on it & I had enough time to spend on it \\
\hline INT10 & I had the need to be helped or trained & I had no need to be helped or trained \\
\hline INT11 & Powerless over my environment & In control of my environment \\
\hline \multicolumn{3}{|c|}{ Technical context } \\
\hline TEC1 & Encountered technical problems & Encountered no technical problems \\
\hline \multicolumn{3}{|c|}{ Task context } \\
\hline TASK1 & I was doing several things simultaneously & I was focusing on the task \\
\hline TASK2 & I was often interrupted & I was never interrupted \\
\hline TASK3 & I focused on the product & I focused on attaining my goals \\
\hline TASK4* & ${ }^{*}$ I felt serious & I felt playful ${ }^{*}$ \\
\hline TASK5 & Having no specific tasks to achieve & Having specific tasks to achieve \\
\hline \multicolumn{3}{|c|}{ Temporal context } \\
\hline TEMP1 & I used the system only once & I am using the system regularly \\
\hline TEMP2 & I spent a short time using the system & I spent a long time using the system \\
\hline TEMP3 & I was interacting at an uncomfortable pace & I was interacting at a comfortable pace \\
\hline TEMP4 & It was not the right moment to use the system & It was the right moment to use the system \\
\hline
\end{tabular}

Items assessing perceived contextual dimensions are indicated in bold. Each item is rated on a 7-points bipolar anchor. Items with an asterisk * were removed from the final version of the scale (see Appendix A).

by Osgood [45]). This choice was made in order to allow for easy comparison between contextual factors and UX-related aspects (as it follows the form of the AttrakDiff scale [19] or User Experience Questionnaire [35], two major UX evaluation tools). The response scales were not labelled, as shown in Appendix A.

The overall structure and internal consistency of our UX Context Scale and subscales has been tested through an online study aimed at assessing users' experiences with the online professional network LinkedIn.

\section{METHOD}

\subsection{Participants and Procedure}

The study was broadcast online on the professional network LinkedIn, and thus involves a non-probabilistic sample. The choice of an online study was made in order to assess the interaction context in an ecological setting, and to collect data on a wide variety of interaction contexts. We explained the participants that the main objective of the study was the evaluation of users' experience with LinkedIn. Considered as part of this overall evaluation, the context 
was not explicitly mentioned in the introduction of the survey and participants were not asked to do a specific task while interacting with the system. Participants gave informed consent and were not compensated for their participation. The questionnaire consisted of three parts: evaluation of the system using the abridged AttrakDiff scale, assessment of objective and subjective contextual factors using the UXCS, and demographic questions (age, gender, country of residence, mother tongue and familiarity with technology). 147 complete answers were collected. Ten participants having declared a score of English proficiency inferior to 5 out of 7 were filtered out of the study as a precise understanding of each item was required for this validation study. The remaining 137 valid answers were quite balanced regarding gender, with respectively 65 men and 72 women. Average age of participants was 38.9 years. Almost all participants declared feeling at ease with technology $(M=6.53$ out of $7 ; 97.8 \%$ having a score higher than 5 out of 7 ).

\subsection{Material}

4.2.1 User Experience Evaluation. First, we asked participants to evaluate their experience with the system using the 10-items abridged AttrakDiff [19] [22]. One item (cheap-premium) was proven unreliable in a previous study [15] and thus not included in our study. The measurement relies on bipolar seven-point anchors. Evaluated system's qualities are Pragmatic Qualities (ATD_PRAG), Hedonic Qualities (ATD_HEDO) and Attractivity (ATD_ATT). The multidimensional structure of the AttrakDiff allows for a distinction between several UX dimensions with regards to the impact of the interaction context.

4.2.2 UX Context Scale. Users were invited to fill out the UXCS. Evaluated contextual dimensions are Physical Context, Social Context, Technical Context, Task Context, and Temporal Context. The experimental version of the UXCS is composed of 12 items related to objective contextual factors and 21 items related to subjective contextual factors (see Table 2).

4.2.3 Data Analysis. Data was analysed using the SPSS Statistics 25. Item codes will be used for the description of the results (see Table 2). There was no missing data. Univariate statistics were run to examine the means and standard deviations of each item as well as to check for possible outliers or entry errors. No outliers or entry errors were found. For the sake of brevity and following APA norms, detailed statistics presented in tables are not necessarily repeated in the text.

\section{RESULTS}

\subsection{Validation of the UX Context Scale}

A principal component factor analysis was run on 21 items initially composing the perceived aspects of the UX Context Scale. The suitability of PCA was assessed prior to analysis. Inspection of the correlation matrix showed that all variables had at least one correlation coefficient greater than 0.3 . The overall Kaiser-MeyerOlkin (KMO) measure was 0.76 , which is 'middling' according to Kaiser's [29] classification and higher to the .6 value suggested by Tabachnick and Fidell [63]. Bartlett's Test of Sphericity was statistically significant $(p<.001)$, indicating that the data was likely factorizable.
Principal component analysis provided an initial number of six possible factors (based on components with eigenvalues greater than 1), explaining $65 \%$ of variance in the data. Three items (SOC5, TASK4, INT5), not loading clearly on any of the six factors identified, were deleted from the scale. The final dataset was composed of 18 pairs of items. Component loadings and communalities of the rotated solution are presented in Table 3 . This six-factor solution explains $70.2 \%$ of variance in the data. A Varimax orthogonal rotation was employed to aid interpretability.

The first factor, related to the Internal context, explains $27 \%$ of the observed variance in the data. The second factor, related to the Physical context, explains $14.8 \%$ of variance, the remaining four factors explaining between 8.8 and $5.7 \%$ of observed variance each. The rotated component matrix shows that almost all items have high loadings on their respective factor and low loadings on all other factors. Exceptions are items INT8 (I had insufficient skills to use it / I had sufficient skills to use it) and INT9 (I did not have enough time to spend on it/I had enough time to spend on it), which overlap on several dimensions. Except for the internal context, which appears to be split up into two different constructs, this six-factors solution reflects a structure that adequately relates to our empirical examination of the interrelationships among contextual-related dimensions. Identified subsets share sufficient variation to justify their existence as factors measuring the perceived context of humancomputer interactions. As shown in Table 3, four items composing the initial Internal context subscale appear to load on a separate subscale (items INT8 to INT11). We call this subscale Perceived resources (RES_CTX), as all four items are dealing with the user's perceived resources in terms of skills, time, need for training or control over the environment. Differentiating Perceived resources from Internal context makes sense if one considers that resources might not only relate to the user's mental state but could also be attributed to an external causality. It is aligned with [3] definition of resource availability.

Reliability of the subscales has been tested using Cronbach's alpha (Table 4) [8]. All subscales showed high internal consistency, with Cronbach's alpha ranging from .68 (Perceived Resources) to .93 (Internal Context). All subscale items are worth retaining as: (1) no single deletion would cause a substantial increase in Cronbach's alpha and (2) all items correlate with their total scale to a moderate or high degree. Overall, the UXCS (including 18 items divided into 6 contextual dimensions) had a Cronbach's alpha $=.78$. We computed a component-based score for each perceived contextual subscale along with a global score for the perceived quality of the context.

The experimental version of the UXCS has been refined according to these results, by splitting the internal context into internal context and perceived resources. Each of this subscale entails 5 items in total when counting both objective and perceived contextual dimensions. The final version of the scale is composed of 30 items (12 items measuring the objective context and 18 items measuring the perceived context) divided into 7 dimensions (the Technical context constitutes a single item dimension, only measuring an objective aspect, thus not included in the preceding factorial and reliability analyses). 
Table 3: Rotated structure matrix for PCA (Varimax rotation)

\begin{tabular}{|c|c|c|c|c|c|c|c|}
\hline \multirow[t]{2}{*}{ Scale / Item } & \multicolumn{6}{|c|}{ Rotated Component Coefficients } & \multirow[t]{2}{*}{ Communalities } \\
\hline & 1 & 2 & 3 & 4 & 5 & 6 & \\
\hline INT3 & .708 & & & & & & .596 \\
\hline INT5 & .801 & & & & & & .766 \\
\hline INT6 & .854 & & & & & & .772 \\
\hline INT7 & .738 & & & & & & .685 \\
\hline PHYS2 & & .644 & & & & & .564 \\
\hline PHYS5 & & .792 & & & & & .693 \\
\hline PHYS6 & & .880 & & & & & .825 \\
\hline INT8 & .482 & & .677 & & & & .730 \\
\hline INT9 & & & .573 & & & .331 & .471 \\
\hline INT10 & & & .774 & & & & .659 \\
\hline INT11 & & & .694 & & & & .672 \\
\hline TASK1 & & & & .694 & & & .596 \\
\hline TASK2 & & & & .793 & & & .772 \\
\hline TASK3 & & & & .794 & & & .679 \\
\hline TEMP3 & & & & & & .803 & .746 \\
\hline TEMP4 & & & & & & .836 & .822 \\
\hline SOC3 & & & & & .848 & & .815 \\
\hline SOC4 & & & & & .833 & & .783 \\
\hline EigenValue & 4.86 & 2.67 & 1.58 & 1.4 & 1.1 & 1 & \\
\hline$\%$ variance explained & 27 & 14.8 & 8.8 & 7.8 & 6.1 & 5.7 & \\
\hline
\end{tabular}

Extraction Method: Principal Component Analysis. Rotation Method: Varimax with Kaiser Normalization. Component loadings <.30 are suppressed. Major loadings for each item are bolded.

Table 4: UXCS subscales: reliability analysis (Cronbach's $\alpha$ )

\begin{tabular}{lllllll}
\hline Subscale & Nb items & Min & Max & M & SD & $\alpha$ \\
\hline PHYS_CTX & 3 & 3 & 7 & 5.88 & 0.93 & .75 \\
SOC_CTX & 2 & 1 & 7 & 4.53 & .25 & .75 \\
INT_CTX & 4 & 2.5 & 7 & 5.31 & 1.07 & .86 \\
RES_CTX & 4 & 1.25 & 7 & 5.70 & 1.02 & .68 \\
TASK_CTX & 3 & 2 & 7 & 4.91 & 1.34 & .69 \\
TEMP_CTX & 2 & 1 & 7 & 5.60 & 1.06 & .74 \\
UXCS_TOTAL & 18 & 3.37 & 7 & 5.32 & 0.65 & .78 \\
\hline
\end{tabular}

\subsection{Perceived Contextual Dimensions}

Participants' ratings (7-points scales) indicated that perceived contextual dimensions were on average positively evaluated (UXCS_TOTAL $M=5.32, S D=0.65$ ) (Table 4). The physical context and perceived resources subscales were assessed as the most positive dimensions ( $M=5.88$ and 5.70, respectively), whereas the social context and task context obtained the lowest ratings $(M=$ 4.53 and 4.91, respectively). Bivariate correlations analyses were performed to study the relationships between perceived contextual subscales (Table 5). The physical context is significantly and positively correlated to all other subscales, except the social context. Main relationships are found between physical context and temporal context ( $r=.31)$, task context ( $r=.31)$ and internal context $(r=.27)$. Feeling pleased by the physical surrounding environment is thus linked to every other context dimension but not to the feeling of relatedness and social support. The social context is mainly linked to the internal context $(r=.42)$. Except for the task context, the internal context is also correlated to all other subscales, especially to the perceived resources $(r=.46)$ and temporal context $(r=.33)$. Perceived 
Table 5: Bivariate correlations between UXCS perceived contextual subscales

\begin{tabular}{|c|c|c|c|c|c|c|c|}
\hline \multicolumn{2}{|c|}{ Perceived Context Subscale } & PHYS & SOC & RES & INT & TASK & TEMP \\
\hline \multirow[t]{2}{*}{ PHYS } & Pearson correlation & 1 & ,128 & $194^{*}$ & $273^{* *}$ &, $306^{* *}$ &, $306^{* *}$ \\
\hline & Sig. (2-tailed) & & ,137 & 023 &, 001 &, 000 &, 000 \\
\hline \multirow[t]{2}{*}{ SOC } & Pearson Correlation & ,128 & 1 &, $189^{*}$ &, $425^{* *}$ &,- 013 &, $189^{*}$ \\
\hline & Sig. (2-tailed) & ,137 & & 027 &, 000 & ,880 & 027 \\
\hline \multirow[t]{2}{*}{ RES } & Pearson Correlation & $194^{*}$ &, $189^{*}$ & 1 &, $456^{* *}$ & 021 &, $345^{* *}$ \\
\hline & Sig. (2-tailed) & 023 & 027 & & 000 & ,810 &, 000 \\
\hline \multirow[t]{2}{*}{ INT } & Pearson Correlation &, $279^{* *}$ &, $448^{* *}$ &, $462^{* *}$ & 1 &,- 086 &, $379^{* *}$ \\
\hline & Sig. (2-tailed) & 001 &, 000 &, 000 & & ,318 &, 000 \\
\hline \multirow[t]{2}{*}{ TASK } & Pearson Correlation &, $306^{* *}$ &,- 013 &, 021 &,- 072 & 1 &, $286^{* *}$ \\
\hline & Sig. (2-tailed) & ,000 & ,880 & ,810 & ,405 & & ,001 \\
\hline \multirow[t]{2}{*}{ TEMP } & Pearson Correlation &, $306^{* *}$ & $189^{*}$ &, $345^{* *}$ &, $333^{* *}$ &, $286^{* *}$ & 1 \\
\hline & Sig. (2-tailed) & ,000 & 027 & ,000 & ,000 & ,001 & \\
\hline
\end{tabular}

resources are mainly correlated to the internal $(r=.46)$ and temporal context $(r=.34)$, but not to the task context.

\subsection{Links between Objective and Perceived Contextual Dimensions}

Our results show links between the objective reported context of use and perceived quality of the context. Regarding the physical environment, items PHYS1, PHYS3, and PHYS4 are positively correlated to the global perceived context $(r=.32, .38$ and .41 respectively, $p<.001)$. The facts of using LinkedIn in a familiar, quiet and steady environment are linked to a better assessment of the perceived context. Item PHYS1 (I was in an unfamiliar place/in a familiar place) is also positively correlated to perceived resources ( $r=.34$, $p<.001$ ), suggesting that users in a familiar place perceive their internal resources as better than users in an unfamiliar place. The correlation between PHYS7 (I was moving/I was remaining still) and TEC1 (technical problems were encountered/no technical problems were encountered $)(r=.45, p<.001)$ suggests that more technical issues were encountered when the user was moving (probably using the mobile version of the system). Results also show that being in a private space (vs. in a public space; item SOC1) is related to a better focus on the task $(r=.31, p<.001)$ and a better assessment of perceived physical context $(r=.39, p<.001)$. On the contrary, SOC2 (I was not interacting with people/interacting with people) is negatively correlated to the task context $(r=-.38, p<.001)$ especially TASK2 $(r=-.48, p<.001)$ : the more users were interacting with people, the less they focused on the task due to interruptions.

The results also show that the more users had specific tasks to achieve, the higher their expectations about the system (correlation between TASK5 and INT1; $r=.31, p<.001)$. Unsurprisingly, the correlation between perceived resources and INT2 $(r=.21, p=.015)$ suggests that having previous experience with the system increases the assessment of perceived internal resources.

\subsection{Impact of Contextual Dimensions on User Experience}

Overall, the user experience of the platform (ATD_TOTAL) is evaluated as quite good with an average score of $M=4.82(S D=0.91)$.
Attractiveness $(M=5.11, S D=1.03)$ and Pragmatic Quality $(M=$ $4.91, S D=1.05)$ are assessed as better than Hedonic Quality $(M=$ $4.51, S D=1.04)$. Table 6 presents descriptive statistics and reliability analyses related to the AttrakDiff scale and subscales.

What is the impact of contextual dimensions on UX? Table 7 shows the bivariate correlations between UXCS and AttrakDiff, with their respective subscales. First, we see that the UXCS is positively correlated to the AttrakDiff $(r=.43)$ and to all its subscales (from .37 to .38). The more the context is perceived as positive, the more the user experience is reported as positive as well. A linear regression performed with UXCS_TOTAL as an independent variable and ATD_TOTAL as a dependant variable show that context properties predicted UX scores, Bêta $=.43, t(136)=5.47, p<.001$, and that $18.1 \%$ of the total variation in the reported UX can be explained by the perceived quality of the context, $R^{2}=.18, F(1,135)=29.8, p$ $<.001$. When only entering INT_TOTAL as independent variable, we see that this variable predicts UX scores, Bêta $=.57, t(136)=$ $10.5, p<.001$, and explains $45 \%$ of the total variation in the reported AttrakDiff rating, $R^{2}=.45, F(1,135)=110.65, p<.001$. Perceived context, and especially Internal context is useful as a predictor of UX assessed by the AttrakDiff scale.

At the subscale level, the main correlation is found between the perceived internal context and the AttrakDiff scale $(r=.67, p$ $<.001)$ and subscales. Perceived internal context is therefore the dimension being the most strongly linked to the global user experience. Regarding the impact of the opinion about the system on the UX, we see a strong correlation between item INT3 and the AttrakDiff $(r=.62, p<.001)$. This confirms previous findings by Raita and Oulasvirta [49], which showed how favourable product expectations boost subjective usability ratings. Both user's motivation (INT5) and interest (INT6) are strongly correlated to the AttrakDiff score ( $r=.55$ and $r=.54$, respectively).

In addition to the internal context subscale, three other subscales are correlated to the AttrakDiff: social context $(r=.33)$, perceived resources $(r=.36)$ and temporal context $(r=.28)$. The correlations are however weaker than the coefficients observed between internal context and UX. Social context is especially linked to the perceived hedonic quality $(r=.31)$ and attractivity $(r=.33)$, whereas Perceived resources is more correlated to the system's pragmatic quality $(r$ 
Table 6: Abridged AttrakDiff scale: descriptive statistics and reliability analyses using Cronbach's alpha $\alpha(\mathrm{N}=137)$

\begin{tabular}{lllll}
\hline & M & SD & Nb items & $\alpha$ \\
\hline AttrakDiff Total (ATD_TOTAL) & 4.82 & 0.91 & 9 & .90 \\
Pragmatic Quality (ATD_PRAG) & 4.91 & 1.05 & 4 & .83 \\
Hedonic Quality (ATD_HEDO) & 4.52 & 1.04 & 3 & .79 \\
Attractivity (ATD_ATT) & 5.11 & 1.03 & 2 & .84 \\
\hline
\end{tabular}

Table 7: Bivariate correlations between UXCS subscales and AttrakDiff $(\mathrm{N}=137)$

\begin{tabular}{llllll}
\hline Perceived Context Subscale & ATD_TOTAL & ATD_PRAG & ATD_HEDO & ATD_ATT \\
\hline UXCS_TOTAL & Pearson Correlation &, $425^{* *}$ &, $368^{* *}$ &, $376^{* *}$ &, $379^{* *}$ \\
& Sig. (2-tailed) &, 000 &, 000 &, 000 &, 000 \\
PHYS_CTX & Pearson Correlation &, 093 &, 103 &, 086 &, 030 \\
& Sig. (2-tailed) &, 279 &, 229 &, 725 \\
SOC_CTX & Pearson Correlation &, $327^{* *}$ &, $248^{* *}$ &, $312^{* *}$ &, $328^{* *}$ \\
& Sig. (2-tailed) &, 000 &, 003 &, 000 &, 000 \\
INT_CTX & Pearson Correlation &, $671^{* *}$ &, $595^{* *}$ &, $556^{* *}$ &, $626^{* *}$ \\
\multirow{2}{*}{ RES_CTX } & Sig. (2-tailed) &, 000 &, 000 &, 000 &, $251^{* *}$ \\
& Pearson Correlation &, $356^{* *}$ &, $379^{* *}$ &, $262^{* *}$ &, 003 \\
TASK_CTX & Sig. (2-tailed) &, 000 &, 000 &, 002 &, 106 \\
TEMP_CTX & Pearson Correlation &,- 155 &,- 161 &, 122 &, 218 \\
& Sig. (2-tailed) &, 071 &, 060 &, $285^{* *}$ &, $251^{* *}$ \\
& Pearson Correlation &, $281^{* *}$ &, $217^{*}$ &, 001 &, 003 \\
\hline
\end{tabular}

$=.38)$ than to its hedonic quality $(r=.26)$ or attractivity $(r=.25)$. The positive correlations between the temporal context and AttrakDiff subscales (ranging from .217 to .285) confirm previous findings by Partala and Kallinen [48], who witnessed a bigger level of time pressure during most unsatisfying experiences compared to most satisfying experiences reported by users. The two remaining UXCS subscales, namely physical context and task context, are not correlated to the global AttrakDiff rating nor to any of its subscales.

\section{DISCUSSION}

\subsection{Development of the UX Context Scale}

Our findings confirm the relevance of the UXCS for the measurement of both objective and perceived contextual factors involved in user experiences. Regarding perceived contextual factors, principal components analysis and reliability analyses have shown the coherence of the current structure of the scale including 18 items divided into 6 contextual dimensions. As compared to the initial envisioned structure, three items were deleted (SOC5, INT5 and TASK4) and four items of the Internal Context subscale that were loading on a separate factor now compose the Perceived Resources subscale (to which we added the accompanying objective context item INT2). All UXCS subscales show a high internal consistency, with Cronbach's alpha ranging from .68 (Perceived Resources) to .86 (Internal Context). Following Grimm and Yarnold's [13] recommendations on the subjects-to-variables ratio, validating the construction of our scale through principal component analyses required a representative sample, sized minimum 5 times bigger than the amount of items used (and a minimum of 100 participants regardless of the ratio). With a sample of 137 participants, we could adequately analyse the results of the initial 21 pairs of items composing the UXCS perceived context. Nevertheless, the validation of a summated rating scale requires the consolidation of results through several studies involving large samples of participants and various use cases.

In the design of the UXCS, the distinction between objective and perceived contextual dimensions allows for a better understanding of the context of use and its impact on the felt experience. Thirteen items from the UXCS aim at informing about the objective contextual situation in which respondents are placed during the interaction with a system. In the case study used to conduct our analyses (on the social network LinkedIn), these items focused on the objective use context and provided us with valuable information. We learned that the interaction generally takes place in a quiet, familiar place and that the users are majorly not interacting with other people. The participants had previous experience with the system. The users declared having some expectations about the system and specific tasks to achieve. This information might seem trivial and one might argue that user research (e.g., interviews or observation) could provide the same results. However, in the case of online studies, practitioners do not necessarily have access to such valuable information to characterize the context of interaction. Sensor data, widely used in pervasive computing [44], can provide a lot of contextual information (on time, activity, movement, device, external elements such as weather, etc) but still faces challenges and fails to understand several aspects of the context. 
In addition, as these 12 items can easily be used as independent variables in statistical analyses, the UXCS allows for the evaluation of the impact of these contextual parameters on UX. We can for instance understand how being in mobility or interacting with people influences the overall experience with a system or service. The resulting 30-items scale, formalized as the User Experience Context Scale (UXCS), is integrative (encompassing all dimensions of the interaction context), multi-dimensional (with independent subscales for each dimensions) and generic (applicable to a wide range of settings and evaluation type). It supports a richer view on the influence of contextual dimensions on user experience.

\subsection{Links between UX and Contextual Dimensions}

Beyond aspects related to the construction of the scale, this study also contributes to exploring the links between UX and the several context dimensions composing the UXCS. In the present study, the overall perceived quality of the interaction context statistically explained $18 \%$ of the variability in UX assessment, thus providing evidence for the relevance of such a measurement.

We saw that the UXCS is positively correlated to the AttrakDiff $(r=.43, p<.001)$ and to all its subscales. The more the context is perceived as positive, the more the user experience is reported as positive as well.

At the subscale level, the internal context, which was already identified as a key dimension in the literature, is significantly and strongly correlated to every AttrakDiff's subscale. This internal context explains $45 \%$ of the total variation in the reported UX score. This is not surprising as UX is focused on the user and their feelings during the interaction. The user is one of the pillars influencing UX, along with the system and the context. Studying the internal context as a distinct dimension may thus seem redundant since the user (and their internal context) is already part of measured UX. This assumption would support Hassenzahl's understanding of the context and the way his research work on UX deals with context. Can a judgment on the appeal of a product be "context-free"[22]? Instead of focusing on the situation (context), he argued to focus on the meta-motivational state the situation triggers, so called "usages modes" [20]. His claim is that these usage modes may be easier to define and more general than the complex description of the situation. In other words, it seems to play no role whether some contextual factors are positive or negative unless this somehow impacts the user's internal state, which in turn impacts product perception and evaluation. This internal context would "summarize" external attributes of the environment. Usage modes were experimentally manipulated in some of his studies $[16,17]$ and a "Usage Mode Scale" was even featured in one of them [21]. Data provided in the present study tend to support Hassenzahl's assumption claiming that what is crucial is the internal state of mind, triggered by the context.

Our results show an absence of correlation between the physical context and the AttrakDiff scale or any of its subscales, suggesting that the physical environment is not linked to the felt experience. In our study, the high average rating and small standard deviation related to the Physical Context subscale $(M=5.88, S D=0.93)$ does not allow for a distinction between users placed in a deteriorated physical environment vs. users placed in a comfortable physical environment. Previous research has shown that characteristics of the physical context such as the physical location or user's mobility affected task performance $[28,42]$. Hence, it seems that different aspects of physical context indeed play a great role in the quality of an interaction, and unfavourable contexts have been compared in the literature to temporary physical or cognitive impairments [2]. This line of research is related to the concept of "situationallyinduced impairments and disabilities" [54, 55]. In this view, users' abilities are constrained by contextual factors (e.g., one-hand operation, vibrations, demands for the user's attention, stress), which temporarily impede reaching an optimal level of performance in the interaction. As these situationally-induced impairments are temporary, the user is less likely to develop a coping strategy. This fact strengthens the need for devices and interfaces that adapt automatically depending on a given physical context [54]. To explain the absence of correlation between physical context and the perceived UX in the present study, our assumption is that the physical context may act as a "deficiency need, that creates negative affect if blocked, but not necessarily strong positive feelings if fulfilled" ([14] p. 358). The physical context would thus negatively impact the UX if perceived as uncomfortable but not necessarily create a positive UX if perceived as comfortable. The same assumption could be relevant to test regarding technical issues a user might encounter during interaction (item TEC1). Not encountering any issue would not be seen as particularly satisfying, whereas encountering technical problems could strongly impede the quality of the interaction and reported UX.

\subsection{Limitations and Future Work}

The present research entails several limitations. First, the UXCS has been tested in the present study on a single sample involving a single use case. As in all scale development processes, it is thus necessary to consolidate these results on additional cases and samples to ensure the stability and validity of the UXCS structure. Further testing the scale in different contexts also allows to avoid the pitfall of UX scales described by [34], who critically reflect on the use and relevance of standardized scales to conduct evaluations in business contexts. To do so, we plan to apply the UXCS to different use cases encompassing various usage modes, and to pay a special attention to the four context properties described by Jumisko-Pyykkö and Vainio [27]. The impacts of the level of magnitude, level of dynamism or type of patterns on the UX should be explored, as well as typical combinations of contextual dimensions (i.e., which different context elements are usually present simultaneously). As the current study design was not adequate to inform on the relation between UX and the physical context, future studies should also focus on differences in the physical context. This can be made for instance by using a between-subjects experimental protocol where a group of users placed in a "deteriorated" physical environment (e.g. noise, mobility) is compared to users placed in a comfortable physical environment. As of now, and beyond simple considerations about the distribution of answers on the scales, it is challenging to speculate on which values indicate an "adequate" or 
"good" perceived context. Some UX scales, like the UEQ [35], provide a benchmark using data from hundreds of product evaluations covering a wide range of applications.

Additionally, the formulation of INT1 "I had no expectations about the systems" vs. "I had high expectations about the system" appears as ambiguous in wording as the opposite of "high" would be "low" and the opposite of "not having expectations" would simply be to "have certain expectations". Consistently with the other items of that scale, the second option could be preferred. Yet, whether the presence of expectations is considered as an objective factor (factual information as per our definition) or a subjective one (tinted by a value judgment) should also be discussed in future work. Raita and Oulasvirta [49] define product expectations as "beliefs and/or emotions related to a product that are formed before its actual use" and further state that the "important of product expectations is related to the fact that even when a technology is novel to its users, certain predispositions still shape their actions and experiences".

Last, the number of items composing the scale overall (30 items) might seems too high to be considered time efficient, especially in survey studies where the UXCS would be combined with other attitudinal measurement items. As every subscale of the perceived context shows high internal reliability, it is possible to use the UXCS in a modular fashion by selecting only the dimensions of the context of interest for each study. As previously discussed, the internal context and perceived resources might for some seems redundant with measurement of the overall user experience, for instance when combined with a measure through the Attrakdiff [19] or UEQ [35] questionnaires as it was the case in the present research protocol. Those two subscales could be filtered out when appropriate.

\section{CONCLUSION}

The development of the User Experience Contextual Scale (UXCS) aims at providing a holistic measurement of contextual factors for UX assessment. The creation of this measurement tool contributes to filling the gaps in UX evaluation questionnaires, which are currently lacking a self-declared measure of the quality of the context. The resulting 30-item scale, formalized as the User Experience Context Scale (UXCS), is integrative (encompassing all dimensions of the interaction context), multi-dimensional (with independent subscales for each dimensions) and generic (applicable to a wide range of settings and evaluation type). It supports a richer view on the influence of contextual dimensions on user experience.

Our findings confirm the relevance of the UXCS as a distinct measure of the context especially useful to control for the influence of the context or to characterize contextual aspects involved in a situation. Being able to isolate the portion of variance due to each contextual dimension is one of the benefits of the UXCS. This study also showed that the format of the UXCS makes it an easy to use, easy to deploy and easy to answer questionnaire. From a psychometrical perspective, the current UXCS structure was confirmed by a PCA showing good internal consistencies of its subscales. Of course, additional use cases involving other experimental situations are needed to provide further empirical validation of our scale. By proposing a quantitative and self-reported measurement tool to assess contextual factors, we aim at easing and promoting the integration of the context as a core dimension of UX evaluations. The UXCS scale can be used both for the design of an interactive system and for its evaluation. In the first case, the focus will be on the process (formative evaluation) allowing product designers to better take into account contextual factors. In the second case, the focus will be on the product itself (summative evaluation), and the UXCS will collect valuable information on the influence of context on the perceived quality of interaction. Both applications might be especially relevant for highly context-dependent products, as for example mobile devices.

\section{ACKNOWLEDGMENTS}

The present project was supported by the Fonds National de

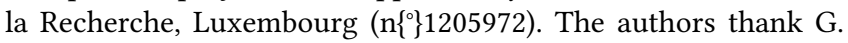
Gronier for his comments on a former version of this paper, as well as the anonymous reviewers whose remarks and requests contributed to improve the quality of the paper.

\section{REFERENCES}

[1] David Alonso-Riós, Ana Vaźquez-Garciá, Eduardo Mosqueira-Rey and Vincente Moret-Bonillo. 2010. A Context-of-Use Taxonomy for Usability Studies. International Journal of Human-Computer Interaction 26, 10, 941-970. http: //dx.doi.org/10.1080/10447318.2010.502099

[2] Leon Barnard, Ji Soo Yi, Julie A. Jacko, and Andrew Sears. 2007. Capturing the effects of context on human performance in mobile computing systems. Personal and Ubiquitous Computing 11, 2 (2007), 81-96. DOI:http://dx.doi.org/10.1007/ s00779-006-0063-x

[3] Christine Bauer and Alexander Novotny. 2017. A consolidated view of context for intelligent systems. Journal of Ambiant Intelligence and Smart Environments, 377-393. DOI:10.3233/AIS-170445

[4] Nigel Bevan and Miles Macleod. 1994. Usability measurement in context. Behaviour \& Information Technology 13, 1-2 (1994), 132-145. DOI:http://dx.doi.org/ $10.1080 / 01449299408914592$

[5] Nicholas A. Bradley and Mark D. Dunlop. 2005. Toward a Multidisciplinary Model of Context to Support Context-Aware Computing. Hum-Comput Interaction 20, 4, 403-446. http://dx.doi.org/10.1207/s15327051hci2004_2

[6] Margaret M. Bradley and Peter J. Lang. 1994. Measuring emotion: The selfassessment manikin and the semantic differential. Journal of Behavior Therapy and Experimental Psychiatry 25, 1 (1994), 49-59. DOI:http://dx.doi.org/10.1016/ 0005-7916(94)90063-9

[7] Nathan Crilly, James Moultrie, and P. John Clarkson. 2004. Seeing things: consumer response to the visual domain in product design. Design Studies 25, 6 (2004), 547-577. DOI:http://dx.doi.org/10.1016/j.destud.2004.03.001

[8] Lee J. Cronbach. 1951. Coefficient alpha and the internal structure of tests. Psychometrika 16, 297-334

[9] Anind K. Dey and Gregory D. Abowd. 2000. Towards a Better Understanding of Context and Context-Awareness. In Proceedings of Workshop on The What, Who, Where, When, and How of Context-Awareness, part of the 2000 Conference on Human Factors in Computing Systems (CHI 2000). ACM, New York, NY.

[10] Anind K. Dey. 2001. Understanding and Using Context. Pers Ubiquit Comput 5, 1, 4-7. http://dx.doi.org/10.1007/s007790170019

[11] Paul Dourish. 2004. What We Talk About When We Talk About Context. Pers Ubiquit Comput 8, 1, 19-30. http://dx.doi.org/10.1007/s00779-003-0253-8

[12] Jodi Forlizzi and Shannon Ford. 2000. The building blocks of experience. Proceedings of the conference on Designing interactive systems processes, practices, methods, and techniques - DIS 00(2000). DOI:http://dx.doi.org/10.1145/347642. 347800

[13] Laurence G. Grimm and Paul R. Yarnold. 1995. Reading and understanding multivariate statistics, Washington, DC: American Psychological Association.

[14] Marc Hassenzahl (2010). Experience Design: Technology for all the right reasons. San Rafael, CA: Morgan \& Claypool.

[15] Marc Hassenzahl, Sarah Diefenbach, and Anja Göritz. 2010. Needs, affect, and interactive products - Facets of user experience. Interacting with Computers 22, 5 (2010), 353-362. DOI:http://dx.doi.org/10.1016/j.intcom.2010.04.002

[16] Marc Hassenzahl, Markus Schöbel, and Tibor Trautmann. 2008. How motivational orientation influences the evaluation and choice of hedonic and pragmatic interactive products: The role of regulatory focus. Interacting with Computers 20, 4-5 (2008), 473-479. DOI:http://dx.doi.org/10.1016/j.intcom.2008.05.001

[17] Marc Hassenzahl and Daniel Ullrich. 2007. To do or not to do: Differences in user experience and retrospective judgments depending on the presence or 
absence of instrumental goals. Interacting with Computers 19, 4 (2007), 429-437. DOI:http://dx.doi.org/10.1016/j.intcom.2007.05.001

[18] Marc Hassenzahl and Noam Tractinsky. 2006. User experience - a research agenda Behaviour \& Information Technology 25, 2 (2006), 91-97. DOI:http://dx.doi.org/ $10.1080 / 01449290500330331$

[19] Marc Hassenzahl, Michael Burmester, and Franz Koller. 2003. AttrakDiff: Ein Fragebogen zur Messung wahrgenommener hedonischer und pragmatischer Qualität. Berichte des German Chapter of the ACM Mensch \& Computer 2003(2003), 187-196. DOI:http://dx.doi.org/10.1007/978-3-322-80058-9_19

[20] Marc Hassenzahl, Kekez, R., \& Burmester, M. 2002. The importance of a software's pragmatic quality depends on usage modes. In H.Luczak, A. E. Cakir, \& G. Cakir (Eds.). In Proceedings of the 6th international conference on Work With Display Units (WWDU 2002) (pp. 275-276). Berlin: ERGONOMIC Institut für Arbeits- und Sozialforschung.

[21] Marc Hassenzahl. 2002. The Effect of Perceived Hedonic Quality on Product Appealingness. International Journal of Human-Computer Interaction 13, 4 (2002), 481-499. DOI:http://dx.doi.org/10.1207/s15327590ijhc1304_07

[22] Marc Hassenzahl, Axel Platz, Michael Burmester, and Katrin Lehner. 2000. Hedonic and ergonomic quality aspects determine a software's appeal. Proceedings of the SIGCHI conference on Human factors in computing systems - CHI 00(2000). DOI:http://dx.doi.org/10.1145/332040.332432

[23] ISO 9241-210:2010 12 15, Ergonomics of human-system interaction - Part 210 Human-centred design for interactive systems, 2010.

[24] ISO 13407:1999, Human-centered design processes for interactive systems, 1999.

[25] ISO 9241-11:1998(E), Ergonomic requirements for office work with visual display terminals (VDTs) - Part 11: Guidance on usability, 1998.

[26] Carroll E. Izard. 1977. Human Emotions. (1977). DOI:http://dx.doi.org/10.1007/ 978-1-4899-2209-0

[27] Satu Jumisko-Pyykkoänd Teija Vainio. 2010. Framing the Context of Use for Mobile Hci. International Journal of Mobile Human Computer Interaction 2, 4, 1-28. http://dx.doi.org/10.4018/jmhci.2010100101

[28] Satu Jumisko-Pyykkö and Miska M. Hannuksela. 2008. Does context matter in quality evaluation of mobile television? Proceedings of the 10th international conference on Human computer interaction with mobile devices and services MobileHCI 08(2008). DOI:http://dx.doi.org/10.1145/1409240.1409248

[29] Henry F. Kaiser. 1974. An index of factorial simplicity. Psychometrika 39, 1 (1974), 31-36. DOI:http://dx.doi.org/10.1007/bf02291575

[30] Wolfgang Kaltz, Jürgen Ziegler, and Steffen Lohmann. 2005. Context-aware Web Engineering: Modeling and Applications. Revue d'intelligence artificielle 19, 3 (January 2005), 439-458. DOI:http://dx.doi.org/10.3166/ria.19.439-458

[31] Hoyoung Kim, Jinwoo Kim, Yeonsoo Lee, Minhee Chae, and Youngwan Choi. 2002. An empirical study of the use contexts and usability problems in mobile Internet. Proceedings of the 35th Annual Hawaii International Conference on System Sciences. DOI:http://dx.doi.org/10.1109/hicss.2002.994090

[32] Hannu Korhonen, Juha Arrasvuori, and Kaisa Väänänen-Vainio-Mattila. 2010 Analysing user experience of personal mobile products through contextual factors. Proceedings of the 9th International Conference on Mobile and Ubiquitous Multimedia - MUM 10(2010). DOI:http://dx.doi.org/10.1145/1899475.1899486

[33] Carine Lallemand, Guillaume Gronier, and Vincent Koenig. 2015. User experience A concept without consensus? Exploring practitioners' perspectives through an international survey. Computers in Human Behavior 43 (2015), 35-48. DOI:http: //dx.doi.org/10.1016/j.chb.2014.10.048

[34] Carine Lallemand, Vincent Koenig. 2017. How could an intranet be like a friend to me?: why standardised UX scales don't always fit. Proceedings of the European Conference on Cognitive Ergonomics ECCE 2017, 9-16.

[35] Bettina Laugwitz, Theo Held, and Martin Schrepp. 2008. Construction and Evaluation of a User Experience Questionnaire. Lecture Notes in Computer Science HCI and Usability for Education and Work(2008), 63-76. DOI:http: //dx.doi.org/10.1007/978-3-540-89350-9_6

[36] Effie L. Law, Virpi Roto, Marc Hassenzahl, Arnold P.o.s. Vermeeren, and Joke Kort. 2009. Understanding, scoping and defining user experience. Proceedings of the 27th international conference on Human factors in computing systems - $\mathrm{CHI}$ 09(2009). DOI:http://dx.doi.org/10.1145/1518701.1518813

[37] John Mccarthy and Peter Wright. 2004. Technology as experience. Interactions 11, 5 (January 2004), 42. DOI:http://dx.doi.org/10.1145/1015530.1015549

[38] Martin Maguire. 2001. Context of Use within usability activities. International Journal of Human-Computer Studies 55, 4 (2001), 453-483. DOI:http://dx.doi.org/ 10.1006/ijhc. 2001.0486

[39] Sascha Mahlke and Manfred Thüring. 2007. Studying antecedents of emotional experiences in interactive contexts. Proceedings of the SIGCHI conference on Human factors in computing systems - CHI 07(2007). DOI:http://dx.doi.org/10. 1145/1240624.1240762

[40] Jonathan Maissel, Andrew Dillon, Martin Maguire, Ralph Rengger and Marian Sweeney. 1991. Context Guidelines Handbook. Teddington, UK: National Physical Laboratory. MUSiC Project Deliverable IF2.2.2. Teddington, UK: National Physical Laboratory.

[41] Michael Minge and Laura Riedel. 2013. meCUE - Ein modularer Fragebogen zur Erfassung des Nutzungserlebens. In: S. Boll, S. Maaß\& R. Malaka (Hrsg.): Mensch und Computer 2013: Interaktive Vielfalt (S. 89-98). Mü̈chen, Oldenbourg Verlag. [42] Terhi Mustonen, Maria Olkkonen, and Jukka Hakkinen. 2004. Examining mobile phone text legibility while walking. Extended abstracts of the 2004 conference on Human factors and computing systems - CHI 04(2004). DOI:http://dx.doi.org/ $10.1145 / 985921.986034$

[43] Lennart E. Nacke, Anders Drachen, Kai Kuikkaniemi, Joerg Niesenhaus, Hannu J. Korhonen, Wouter M. van den Hoogen, Karolien Poels, Wijnand A. IJsselsteijn, and Yvonne de Kort. 2009. Playability and Player Experience Research. DiGRA 2009, Breaking New Ground: Innovation in Games, Play, Practice and Theory, Brunel University, United Kingdom.

[44] Alexander Novotny and Christine Bauer. 2017. What Do We Really Talk About When We Talk About Context in Pervasive Computing: A Review and Exploratory Analysis. In Proceedings of 19th International Conference on Information Integration and Web-based Applications \& Services, Salz- burg, Austria, December 4-6, 2017 (iiWAS '17), 10 pages. https://doi.org/10.1145/3151759.3151760

[45] Charles E. Osgood, George J. Suci, and Percy H. Tannenbaum. 1957. The Measurement of Meaning. Chicago: University of Illinois Press(1957).

[46] Antti Oulasvirta, Sakari Tamminen, Virpi Roto, and Jaana Kuorelahti. 2005. Interaction in 4-second bursts. Proceedings of the SIGCHI conference on Human factors in computing systems - CHI 05(2005). DOI:http://dx.doi.org/10.1145/ 1054972.1055101

[47] Antti Oulasvirta. 2008. Field Experiments in HCI: Promises and Challenges. Future Interaction Design II (2008), 87-116. DOI:http://dx.doi.org/10.1007/978-1-84800385-9_5

[48] Timo Partala and Aleksi Kallinen. 2012. Understanding the most satisfying and unsatisfying user experiences: Emotions, psychological needs, and context. Interacting with Computers 24, 1 (2012), 25-34. DOI:http://dx.doi.org/10.1016/j. intcom.2011.10.001

[49] Eeva Raita and Antti Oulasvirta. 2011. Too good to be bad: Favorable product expectations boost subjective usability ratings. Interacting with Computers 23,4 (2011), 363-371. DOI:http://dx.doi.org/10.1016/j.intcom.2011.04.002

[50] Virpi Roto, Effie Law, Arnold Vermeeren, and Jettie Hoonhout. 2011. User Experience White Paper: Bringing clarity to the concept of user experience. Result from Dagstuhl Seminar on Demarcating User Experience, Finland.

[51] Virpi Roto, Web browsing on mobile phones: Characteristics of user experience, Helsinki University of Technology, 2006.

[52] James A. Russell, Anna Weiss, and Gerald A. Mendelsohn. 1989. Affect Grid: A single-item scale of pleasure and arousal. Journal of Personality and Social Psychology 57, 3 (1989), 493-502. DOI:http://dx.doi.org/10.1037/0022-3514.57.3. 493

[53] Richard M. Ryan. 1982. Control and information in the intrapersonal sphere: An extension of cognitive evaluation theory. Journal of Personality and Social Psychology 43, 3 (1982), 450-461. DOI:http://dx.doi.org/10.1037//0022-3514.43.3. 450

[54] Andrew Sears, Min Lin, Julie Jacko and Yan Xiao. 2003. When computers fade. . pervasive computing and situationally-induced impairments and disabilities, in: Human-Computer Interaction: Theory and Practice, Vol. 2, C. Stephanidis and J.A. Jacko, eds, 2003, pp. 1298-1302.

[55] Andrew Sears, Mark Young, and Jinjuan Feng. 2007. Physical Disabilities and Computing Technologies. The Human-Computer Interaction Handbook Human Factors and Ergonomics (2007), 829-852. DOI:http://dx.doi.org/10.1201/ 9781410615862.ch42

[56] Nora C. Schaeffer and Stanley Presser. 2003. The Science of Asking Questions. Annual Review of Sociology 29, 1 (2003), 65-88. DOI:http://dx.doi.org/10.1146/ annurev.soc.29.110702.110112

[57] Klaus R. Scherer. 2001. Appraisal considered as a process of multi-level sequential checking. In K. R. Scherer, A. Schorr, \& T. Johnstone (Eds.), Appraisal processes in emotion: Theory, methods, research (pp. 92-120). New York: Oxford University Press.

[58] Bill N. Schilit, Norman Adams and Roy Want. 1994. Context-aware computing applications, in: Proc. 1st Workshop on Mobile Computing Systems and Applications (WMCSA 1994), IEEE, 1994, pp. 85- 90

[59] Kennon M. Sheldon, Andrew J. Elliot, Youngmee Kim, and Tim Kasser. 2001. What is satisfying about satisfying events? Testing 10 candidate psychological needs. Journal of Personality and Social Psychology 80, 2 (2001), 325-339. DOI:http: //dx.doi.org/10.1037/0022-3514.80.2.325

[60] Paul E. Spector. 1992. Summated rating scale construction: an introduction. Newbury Park, CA: Sage Publisher.

[61] Jared M. Spool. 2005. Putting Context Into Context [Web blog post]. Retrieved from https://articles.uie.com/putting_context_into_context/

[62] Lucy Suchman. 1987. Plans and Situated Actions: the Problem of Human-Machine Communication. New York: Cambridge University Press

[63] Barbara G. Tabachnick and Linda S. Fidell. 2001. Using multivariate statistics, Boston: Allyn and Bacon.

[64] Alexandre N. Tuch, Rune Trusell, and Kasper Hornbæk. 2013. Analyzing users narratives to understand experience with interactive products. Proceedings of the SIGCHI Conference on Human Factors in Computing Systems - CHI 13(2013). DOI:http://dx.doi.org/10.1145/2470654.2481285 
[65] Katarzyna Wac, Selim Ickin, Jin-Hyuk Hong, Lucjan Janowski, Markus Fiedler, and Anind K. Dey. 2011. Studying the experience of mobile applications used in different contexts of daily life. Proceedings of the first ACM SIGCOMM workshop on Measurements up the stack - W-MUST 11(2011). DOI:http://dx.doi.org/10.1145/ 2018602.2018605

[66] David Watson, Lee A. Clark, and Auke Tellegen. 1988. Development and validation of brief measures of positive and negative affect: The PANAS scales. Journal of Personality and Social Psychology 54, 6 (1988), 1063-1070. DOI:http://dx.doi.org/ 10.1037//0022-3514.54.6.1063

[67] Heli Wigelius and Heli Vaätaja: 2009. Dimensions of context affecting user experience in mobile work, in: Proc. INTERACT 2009, Springer, 2009, pp. 604-617.

\section{A APPENDICES}

\section{A USER EXPERIENCE CONTEXT SCALE - FINAL VERSION}

The UXCS is composed of 30 items in total: 12 items assessing objective contextual dimensions and 18 items assessing perceived contextual dimensions (in bold). Each item is rated on a 7-points bipolar anchor.

While using the system, I was:

Physical context

\begin{abstract}
In an unfamiliar place
In an unpleasant location

In a noisy place

Unpleased with the lighting
\end{abstract}

In a moving / vibrating environment

Unpleased with the temperature

Moving (mobile usage)

Social context

$$
\begin{array}{r}
\text { In a public space } \\
\text { Not interacting with people } \\
\text { Feeling alone }
\end{array}
$$

Feeling unsupported

Task context

Doing several things simultaneously Often interrupted

Focused on the product

Having no specific tasks to achieve

Technical context

Encountering technical problems

Internal contex

Demotivated
Not interested
In a bad mood

While using the system, (I had):

Internal context

No expectations about the system A bad opinion about it

Perceived resources

No previous experience with the system Insufficient skills to use it

Not have enough time to spend on it

The need to be helped or trained

Powerless over my environment

Temporal context

Used the system only once Spent a short time using the system

I was interacting at an uncomfortable pace It was not the right moment to use the system
0000000

0000000

0000000

0000000

0000000

$0 \bigcirc \bigcirc \bigcirc \bigcirc \bigcirc \bigcirc$

0000000

0000000

0000000

0000000

0000000

0000000

0000000

0000000

0000000

0000000

$0 \bigcirc \bigcirc \bigcirc \circ \bigcirc \bigcirc$

0000000

0000000

0000000

0000000

$0 \bigcirc \bigcirc \bigcirc \bigcirc \bigcirc \bigcirc$

0000000

$000 \bigcirc \bigcirc \bigcirc 0$

0000000

0000000

0000000 0000000

$0 \bigcirc 00000$

0000000
In a familiar place

In a pleasant location

In a quiet place

In a steady environment

Pleased with the temperature

Pleased with the lighting

Remaining still

In a private space

Interacting with people

Feeling related to other people

Feeling supported

Focusing on the task

Never interrupted

Focused on attaining my goals

Having specific tasks to achieve

Encountering no technical problems

Motivated

Interested

In a good mood

High expectations about the system

A good opinion about it

Already some experience with the system

Sufficient skills to use it

Enough time to spend on it

No need to be helped or trained

In control of my environment

Been using the system regularly Spent a long time using the system

I was interacting at a comfortable pace

It was the right moment to use the system 